\title{
内蒙古草甸草原 $\mathrm{CH}_{4}$ 和 $\mathrm{N}_{2} \mathrm{O}$ 排放通量的时间变异
}

\section{王艳芬 ${ }^{1}$ 马秀枝 ${ }^{1}$ 纪宝明 ${ }^{1}$ 杜 睿 $^{2} \quad$ 陈佐忠 ${ }^{1}$ 王庚辰 $^{2}$ 王跃思 $^{2}$ 万小伟 ${ }^{2}$ \\ （1 中国科学院植物研究所, 北京 100093）（2 中国科学院大气物理研究所, 北京 100029）}

\begin{abstract}
摘 要 1998 年 6 月 1 日、7 月 2 日、8 月 3 日和 9 月 1 日用静态箱-气相色谱法对内蒙古锡林河流域草甸草原 $\mathrm{CH}_{4}$ 和 $\mathrm{N}_{2} \mathrm{O}$ 排放通量的昼夜观测表明, 天然草原是 $\mathrm{N}_{2} \mathrm{O}$ 的源、 $\mathrm{CH}_{4}$ 的汇, 其排放和吸收具有明显的昼夜变化规律。4 次 昼夜观测的结果还表明, 草甸草原排放 $\mathrm{N}_{2} \mathrm{O}$ 和吸收 $\mathrm{CH}_{4}$ 的能力具有强烈的季节变化规律。方差分析表明, 不同季

节 $\mathrm{N}_{2} \mathrm{O}$ 排放通量差异显著 $(F=6.359, p<0.004)$, 日均通量变化范围为 $0.282 \sim 2.134 \mu \mathrm{g} \mathrm{N}_{2} \mathrm{O}-\mathrm{N}^{\cdot} \cdot \mathrm{m}^{-2} \cdot \mathrm{h}^{-1}$; 而 $\mathrm{CH}_{4} 4$ 次测定的日均吸收通量分别为 $(-52.19 \pm 19.67) 、(-27.20 \pm 10.57) 、(-126.05 \pm 9.32)$ 和 $(-16.45 \pm 1.31) \mu \mathrm{g} \mathrm{CH}_{4}-$ $\mathrm{C} \cdot \mathrm{m}^{-2} \cdot \mathrm{h}^{-1}$, 从整个生长季看表现为“高-低-高-低”的特点。锡林河流域选择 9:00 13:00 作为采样日起始采样时 间, 并以该时段观测获得的温室气体地-气交换通量来代表当日通量是比较合适的。
\end{abstract}

关键词 $\mathrm{N}_{2} \mathrm{O} \quad \mathrm{CH}_{4}$ 日变化 草甸草原 锡林河流域 内蒙古

\section{DIURNAL AND SEASONAL VARIATION IN METHANE AND NITROUS OXIDE FLUXES IN MEADOW STEPPE OF INNER MONGOLIA}

\author{
WANG Yan-Fen ${ }^{1}$ MA Xiu-Zhi ${ }^{1} \quad$ JI Bao-Ming ${ }^{1} \quad$ DU Rui $^{2} \quad$ CHEN Zuo-Zhong ${ }^{1}$ \\ WANG Geng-Chen ${ }^{2}$ WANG Yue-Si ${ }^{2}$ and WAN Xiao-Wei ${ }^{2}$ \\ (1 Institute of Botany, the Chinese Academy of Sciences, Beijing 100093, China) \\ (2 Institute of Atmospheric Physics, the Chinese Academy of Sciences, Beijing 100029, China)
}

\begin{abstract}
Temperate and tropical oxic soils usually exhibit low levels of atmospheric $\mathrm{CH}_{4}$ oxidation, which are estimated to consume about $10 \%$ of the atmospheric $\mathrm{CH}_{4}$. It has been reported that the semi-arid grasslands represent a significant global sink for $\mathrm{CH}_{4}$ and a source of atmospheric $\mathrm{N}_{2} \mathrm{O}$. However, the spatial and temporal variability of methane is usually very high which may result in the uncertainty of the ecosystem's function in the exchange of greenhouse between ecosystem and atmosphere. In order to help define the possible range of emission of $\mathrm{N}_{2} \mathrm{O}$ and $\mathrm{CH}_{4}$ from temperate grassland of Inner Mongolia, diurnal variation in fluxes of methane and nitrous oxide in meadow steppe in the Xilin River basin were investigated with static-chamber and GC methods on June 1, July 2, August 3 and September 1 of 1998. Suitable sampling time was also discussed with the data of diurnal variation investigation. The native meadow steppe can act as a source of atmospheric $\mathrm{N}_{2} \mathrm{O}$ but a sink of $\mathrm{CH}_{4}$ in which the fluxes of the two greenhouse gases had obvious diurnal variation. ANOVA results showed that there was significant difference for the fluxes of $\mathrm{N}_{2} \mathrm{O}$ in the four months $(F=6.359, p<0.004)$, ranging between $0.282-2.134 \mu \mathrm{g} \mathrm{N} \mathrm{N}_{2} \mathrm{O}-\mathrm{N} \cdot \mathrm{m}^{-2} \bullet \mathrm{h}^{-1}$. These background emission rates were typically similar to those in other temperate grasslands. The diurnal mean uptake rates of methane were $(-52.19 \pm 19.67),(-27.20$ $\pm 10.57)$, $(-126.05 \pm 9.32)$ and $(-16.45 \pm 1.31) \mu \mathrm{g} \mathrm{CH} \mathrm{CH}_{4}-\mathrm{C} \cdot \mathrm{m}^{-2} \cdot \mathrm{h}^{-1}$ for the four month respectively, which has a trend of "high-low-high-low" from the end of May through the beginning of September. The methane uptake rates were similar to those in the Colorado short-grass steppe, averaging $31.2 \mu \mathrm{g} \mathrm{CH}_{4}-\mathrm{C} \cdot \mathrm{m}^{-2}$ $\cdot \mathrm{h}^{-1}$, compared with varieties of temperate ecosystems. It was proved that selecting 9:00 - 13:00 as sampling time for each sampling day is suitable in Xilin River basin according to the diurnal variation measurement.
\end{abstract}

Key words $\mathrm{N}_{2} \mathrm{O}, \mathrm{CH}_{4}$, Diurnal variation, Meadow steppe, Xilin River basin, Inner Mongolia

随着全球变化研究的升温, 人类活动干扰下陆 地生态系统温室气体的源汇研究越来越受到关注, $\mathrm{N}_{2} \mathrm{O}$ 和 $\mathrm{CH}_{4}$ 作为两种重要的温室气体对全球变暖的 贡献约为 $20 \%$, 而且过去几十年中这两种气体的浓
度一直在不断增加 (Mosier et al., 1991), 许多研究 发现人类的农牧业生产活动是造成大气中温室气体 变化的一个重要原因, 例如草地的农牧业利用 (放 牧、农垦、灌溉、施肥等) 增加了土壤-植被系统排放 
$\mathrm{N}_{2} \mathrm{O}$ 的能力, 同时降低了吸收 $\mathrm{CH}_{4}$ 的能力 (Bronson et al ., 1992; Bronson \& Mosier, 1993; Mosier et al ., 1996; Groffman \& Turner, 1995; Smart et al., 1999; Corre et al., 1999; Luo et al., 1999), 改变了草地生 态系统在大气 $\mathrm{N}_{2} \mathrm{O} 、 \mathrm{CH}_{4}$ 平衡中的地位和作用。然 而, 尽管草地生态系统地-气交换通量特征已取得了 许多野外原位观测结果, 要全面评价全球草地生态 系统对全球大气的贡献仍旧十分困难, 研究方法及 历史资料可比性差是其中的一个方面, 更重要的是 大部分已有的观测工作集中在北美、欧洲、澳洲等 地,而其它地区这方面的工作相对滞后。中国拥有 占世界草地面积的 $12.5 \%$ 的草地, 草地生态系统温 室气体研究起步却非常晚(王艳芬等, 1998; 杜睿等, 1998; 王庚辰等, 1998; 王跃思等, 2000; 纪宝明等, 2001）。中国草地中,有许多地区由于其独特的地理 环境和景观特征被认为是全球变化研究的关键地 区, 内蒙古草原便是其中之一。

内蒙古草原是欧亚大陆草原的重要组成部分, 也是目前温带地区保存较好的天然草原之一。受水 分和温度驱动, 自东向西, 依次分布着草甸草原、典 型草原和荒漠草原。草甸草原作为锡林河流域一个 重要的草原类型, 其土壤-植被系统与大气间温室气 体交换通量及其特征, 是判定锡林河流域草原对全 球温室气体贡献的重要指标之一, 草甸草原是一种 地带性植被类型, 可以由锡林河流域外推至内蒙古 草原, 并最终定量给出内蒙古草甸草原在人类活动 干扰下对大气 $\mathrm{CH}_{4} 、 \mathrm{~N}_{2} \mathrm{O}$ 的贡献, 为今后该地区农牧 业产业结构的调整以及社会、经济、生态环境的良性 发展提供科学的决策依据。

本文主要探讨草甸草原 $\mathrm{CH}_{4} 、 \mathrm{~N}_{2} \mathrm{O}$ 排放通量的 昼夜、季节变化特征。

\section{1 研究地点}

锡林河流域 $\left(43^{\circ} 26^{\prime} \sim 44^{\circ} 39^{\prime} \mathrm{N}, 115^{\circ} 32^{\prime} \sim 117^{\circ} 12^{\prime}\right.$ E)地处内蒙古高原东部, 锡林郭勒高平原的东部边 缘。地势从东南向西北逐渐降低, 东部低山最高海 拔 $1506 \mathrm{~m}$, 西北锡林河下游最低处为 $902 \mathrm{~m}$, 全流域 相对高差 $600 \mathrm{~m}$, 总面积 $10786 \mathrm{~km}^{2}$ 。该区地形以低 山丘陵为特征，坡度较缓，低山相对高差不大于 $300 \mathrm{~m}$, 丘陵相对起伏 $30 \sim 150 \mathrm{~m}$ 。在实验区中还有 锡林河及支流之间的风成沙带，宽度最大达 $10 \mathrm{~km}$, 沙丘相对起伏约为 $10 \sim 20 \mathrm{~m}$, 具有中等复杂性的地 形地貌。该区草原原始面貌保存较好, 在气候、植 被、动物和土壤等方面, 不仅在中国温带草原区具有
较强的典型性, 而且对整个欧亚大陆草原区来讲也 有明显的代表性。

研究区内草甸草原是以贝加尔针茅 (Stipa baicalensis) 为建群种的草原群落类型, 这类草原每平 方米有植物种 $29 \sim 39$ 种, 贝加尔针茅、麻花头 ( Serratula centauroides)、线叶菊 (Filifolium sibiricum)、射干 岛尾 (Iris dichotoma) 等中生、旱中生和中旱生植物为 主, 占 $53.3 \%$, 植物群落总盖度为 $63 \%$ 。地上生物 量为 $96 \sim 236 \mathrm{~g} \bullet \mathrm{m}^{-2}$, 地下生物量为 $906 \sim 3254 \mathrm{~g} \bullet$ $\mathrm{m}^{-2}$, 二者的比值为 $9.4 \sim 15.5$ 。该类型草原下发育 的是黑钙土, 其腐殖质层厚度为 $50 \mathrm{~cm}$ 左右; 有机质 含量达 $4 \%$; 碳酸钻淀积不明显或只形成假菌丝体, 没有明显的钲积层。研究地点描述及采样点土壤主 要特征详见文献(王艳芬等, 1998)。

\section{2 研究方法}

\section{1 采样方法}

本研究采用透明静态箱-气相色谱法观测 $\mathrm{CH}_{4}$ 、 $\mathrm{N}_{2} \mathrm{O}$ 的地-气交换通量。采样分析系统主要由采样 箱、样品采集系统、气相色谱分析系统及色谱工作站 组成。采样箱截面为正方形, 边长 $60 \mathrm{~cm}$, 箱高 20 $\mathrm{cm}$, 描述详见文献(王跃思等, 2000)。其基座的安装 深度与采样点土质状况有关, 草原土壤表层较为坚 硬,采样箱基座插入土壤不必太深。将采样箱基座 嵌入采样位点, 使基座上口与地面水平尽量不扰动 观测点的自然状态。一般要在正式采样前一周左右 的时间将采样箱基座安装在采样点, 不破坏原有植 被, 以便使人为扰动对微量气体交换速率观测的影 响降到最低。所测结果为土壤-植被亚系统与大气 之间的气体交换通量。

进行采样时, 首先将箱体罩在已预先安好的基 座上, 同时开始记时并采集第一个样品。将注射器 与箱体一侧的三通阀相联取气体样品, 取样量一般 为 $30 \sim 60 \mathrm{ml}$, 同时记录箱体内温度值 (空气温度、土 壤表面温度和土壤 $10 \mathrm{~cm}$ 处温度); 之后每隔 $20 \mathrm{~min}$ 取样 1 次, 共取样 4 次。采样结束后, 将箱体移走, 样品带回实验室进行分析。样品分析仪器为 HP5890II 型气相色谱, 方法详见文献 (纪宝明等, 2001)。

于 1998 年的 6 月 1 日、7 月 1 日、8 月 2 日和 9 月 1 日对草甸草原进行昼夜变化规律观测。 $24 \mathrm{~h}$ 内 每 $3 \mathrm{~h}$ 采样 1 次, 同时测定箱体内外温度 (气温、地 表温度和土壤 $10 \mathrm{~cm}$ 温度)、土壤湿度和空气湿度。

2.2 计算方法 
气体交换通量计算采用如下公式:

$F=\frac{\Delta m}{A \cdot \Delta t}=\frac{\rho \cdot V \cdot \Delta c}{A \cdot \Delta t}=\rho \cdot H \cdot \frac{\Delta c}{\Delta t}$ 式中, $F$ 为测定气体的交换通量, $\rho$ 为箱内气体密 度, $\Delta m$ 和 $\Delta c$ 分别为 $\Delta t$ 时间内箱内气体质量和混合 比浓度的变化, $A 、 V 、 H$ 分别为采样箱底面积、体积 和气室高度, $\Delta c / \Delta t$ 为箱内气体浓度变化。 $F$ 为负 值时表示吸收, 为正值时表示排放。具体计算时考 虑对气体通量进行气压和温度校正。

\section{3 结果与讨论}

\section{1 天然草甸草原 $\mathrm{N}_{2} \mathrm{O}$ 排放通量昼夜变化规律}

天然草原 $\mathrm{N}_{2} \mathrm{O}$ 排放通量昼夜观测结果表明每 日上午变化较大, 尤其是早上 7:00 左右, 午后出现 一个通量低谷, 之后又有不同程度的上升(图 1)。 从生长物候期来看, $6 \sim 8$ 月 3 个月昼夜通量变化规 律较为一致, 但其通量范围是不同的, 方差分析表 明,4 次测定 $\mathrm{N}_{2} \mathrm{O}$ 排放通量差异显著 $(F=6.359$, $p<0.004$ )。从 4 次观测结果 (表 1) 来看, 8 月 3 日 日均通量最大, 为 $2.134 \mu \mathrm{gN} \cdot \mathrm{m}^{-2} \cdot \mathrm{h}^{-1}, 6$ 月 1 日通 量最低, 仅为 $0.282 \mu \mathrm{gN} \cdot \mathrm{m}^{-2} \cdot \mathrm{h}^{-1}$ 。该结果可能与 1998 年的天气条件有关系, 1998 年是当地少有的丰 水年, 根据中国科学院内蒙古草原生态系统定位研 究站(距采样点 $20 \mathrm{~km}$ ) 的气象记录, 当年总降水量 达 $507 \mathrm{~mm}$, 远远高于当地平均降水量, 而且 7 月降 雨约占全年降雨量的 $41 \%$, 进一步的研究发现, 观

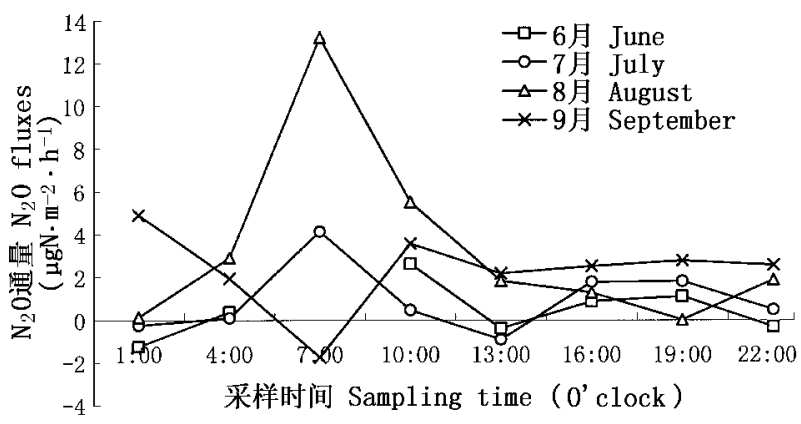

图 1 草甸草原 $\mathrm{N}_{2} \mathrm{O}$ 排放通量昼夜变化 (1998)

Fig. 1 Diurnal variation of $\mathrm{N}_{2} \mathrm{O}$ fluxes in meadow steppe in 1998

表 $1 \mathrm{~N}_{2} \mathrm{O}$ 日平均通量及其变异性

Table 1 Diurnal mean fluxes (DMF) and $C V$ of $\mathrm{N}_{2} \mathrm{O}$

\begin{tabular}{cccc}
\hline \multirow{2}{*}{$\begin{array}{c}\text { 取样时间 } \\
\text { Sampling date } \\
(\text { 日/月 Day/month })\end{array}$} & \multicolumn{3}{c}{ 日平均通量 $\mathrm{DMF}\left(\mu \mathrm{g} \mathrm{N} \mathrm{N}_{2} \mathrm{O}-\mathrm{N}^{\bullet} \mathrm{m}^{-2} \cdot \mathrm{h}^{-1}\right)$} \\
\cline { 2 - 4 } & Mean flux & 均方差 & 变异系数 \\
$\mathrm{STD}$ & $C V(\%)$ \\
\hline $1 / 6$ & 0.282 & 0.802 & 284 \\
$2 / 7$ & 0.613 & 1.011 & 165 \\
$3 / 8$ & 2.134 & 2.766 & 130 \\
$2 / 9$ & 1.492 & 1.209 & 81 \\
\hline
\end{tabular}

STD: Standard square deviation CV: Coefficient of variation
测前三天累计降雨量以 8 月取样最大, 为 $21.3 \mathrm{~mm}$, 其它 3 个月分别为 $1.9 、 2.0 、 1.0 \mathrm{~mm}$, 而 4 次取样的 日均气温变化于 $13.6 \sim 18.4{ }^{\circ} \mathrm{C}$ 之间, 因此土壤水分 状况的变化是 8 月 3 日出现高 $\mathrm{N}_{2} \mathrm{O}$ 通量的可能原 因。在累积降雨量变化不大的 3 次取样中, 6 月 1 日的土壤温度 $(10 \mathrm{~cm})$ 最低, 这可能是 6 月 1 日 $\mathrm{N}_{2} \mathrm{O}$ 通量低的主要原因。由于 $\mathrm{N}_{2} \mathrm{O}$ 主要产生于土壤硝 化过程和反硝化过程 (Williams et al., 1992), 而这 两个过程不仅受土壤理化性质 (土壤有机质含量、 $\mathrm{pH}$ 、土壤容重、土壤质地等) 的影响而具有强烈的空 间变异性, 而且受土壤温度、土壤湿度、 $\mathrm{O}_{2}$ 及碳氮底 物供应能力、植物生长状况等的影响而具有明显的 时间变异性, 所以,在同一采样点进行的不同季节的 观测结果的差异可以简单地表明 $\mathrm{N}_{2} \mathrm{O}$ 通量的时间 变异性。通量的昼夜变化范围很大, 变异系数均在 $80 \%$ 以上。

分析 $\mathrm{N}_{2} \mathrm{O}$ 昼夜通量与大气温度、大气湿度、箱 内空气温度及土壤 $10 \mathrm{~cm}$ 处温度的相关性, 发现 6 月 1 日与 8 月 3 日 $\mathrm{N}_{2} \mathrm{O}$ 排放通量与箱内空气温度存 在较好的正相关关系 $(R=0.564, n=6 ; R=0.563$, $n=8)$ 。

在昼夜规律观测中还有一个值得注意的是负通 量的出现。除了 8 月 3 日, 其它 3 次均观测到了负 通量, 其中 6 月 1 日出现了 3 次, 7 月 2 日出现了两 次, 9 月 1 日 1 次。在这 6 次负通量中, 夜间的 4 次 分别在 $22: 00$ (1 次)、1:00(两次)和 7:30(1 次); 白天 的两次均出现在 $13: 00$ 。由于最大负通量 (绝对值) 仅为 $1.109 \mu \mathrm{g} \mathrm{N} 2 \mathrm{O}-\mathrm{N} \cdot \mathrm{m}^{-2} \cdot \mathrm{h}^{-1}$, 出现的时间也没有 规律, 对其可能产生的机理和生态学意义目前还不 能做出判断。

\section{2 天然草甸草原 $\mathrm{CH}_{4}$ 吸收通量昼夜变化规律}

草甸草原 $\mathrm{CH}_{4}$ 吸收昼夜变化的重要特征体现 在日变幅 (图 2) 上。除 9 月初 (14.19 17.75 $\mu \mathrm{g}$ $\left.\mathrm{CH}_{4}-\mathrm{C} \cdot \mathrm{m}^{-2} \cdot \mathrm{h}^{-1}\right)$ 以外, 其它 3 个月日变幅较大, 分

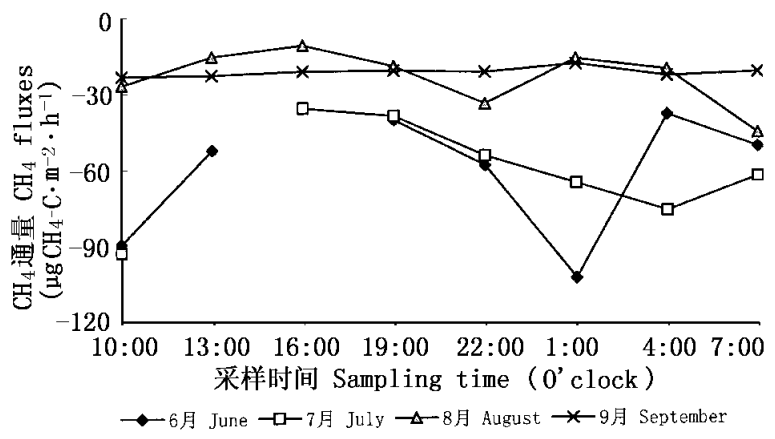

图 2 草甸草原 $\mathrm{CH}_{4}$ 吸收通量昼夜变化

Fig.2 Diurnal change of $\mathrm{CH}_{4}$ fluxes in meadow steppe 
别为 $30.50 \sim 77.44 、 26.98 \sim 69.63$ 和 $8.48 \sim 34.74$ $\mu \mathrm{g} \mathrm{CH}_{4}-\mathrm{C} \cdot \mathrm{m}^{-2} \cdot \mathrm{h}^{-1}$ 。日最大吸收通量均出现在 16:00至次日凌晨 1:00 之间, 而且除 9 月初外, 其它 3 个月的昼夜观测结果均发现有两个吸收高峰: 6 月 初峰值出现在 $16: 00$ 和次日凌晨 $1: 00,7$ 月初峰值 出现在 19:00 和次日 1:00,8 月初则有所提前, 出现 在 $13: 00$ 和 $22: 00$ 。

分析 $\mathrm{CH}_{4}$ 吸收通量昼夜变化与大气温度、大气 湿度、箱内空气温度及箱内土壤 $10 \mathrm{~cm}$ 处温度的关 系, 发现除 9 月 1 日的观测中 $\mathrm{CH}_{4}$ 吸收通量与大气 温度、箱内空气温度及大气湿度存在显著相关关系 之外, 其它月份 $\mathrm{CH}_{4}$ 通量与其它因子之间没有明显 相关关系; $\mathrm{CH}_{4}$ 通量与土壤 $10 \mathrm{~cm}$ 处温度之间没有 明显相关关系。这与董云社等 (2000) 在同一地点获 得的 $\mathrm{CH}_{4}$ 通量与土壤 $5 \mathrm{~cm}$ 处温度的相关性有所不 同, 说明不同层次的土壤温度对土壤吸收 $\mathrm{CH}_{4}$ 的影 响存在差异, 由此也说明目前对草甸草原土壤吸收 $\mathrm{CH}_{4}$ 的机理尚需进一步的实验研究。

4 次昼夜观测结果表明, 草甸草原吸收 $\mathrm{CH}_{4}$ 的 能力具有强烈的季节变化规律, $6 \sim 9$ 月的月初 $\mathrm{CH}_{4}$ 日均吸收通量分别为 $(-52.19 \pm 19.67) 、(-27.20$ $\pm 10.57) 、(-12.05 \pm 9.32)$ 和 $(-16.45 \pm 1.31) \mu \mathrm{g}$ $\mathrm{CH}_{4}-\mathrm{C} \cdot \mathrm{m}^{-2} \cdot \mathrm{h}^{-1}$ 。

$3.3 \mathrm{~N}_{2} \mathrm{O}$ 与 $\mathrm{CH}_{4}$ 通量的关系

对 $\mathrm{N}_{2} \mathrm{O}$ 排放通量与 $\mathrm{CH}_{4}$ 吸收通量所作的回归 分析 (图 3) 表明, 二者具有一定的相关关系 $\left(R^{2}=\right.$ $0.2699, n=24$ ), 此结果与 Mosier 等(1991)在科罗 拉多矮草草原所做的结果是一致的。这个现象与土 壤吸收 $\mathrm{CH}_{4}$ 的生物过程有关, 土壤氧化大气中的 $\mathrm{CH}_{4}$ 主要由甲烷专性氧化酶( MMO) 催化完成 (Davidson \& Schimel, 1995; Conrad, 1996; Topp \& Pattey,

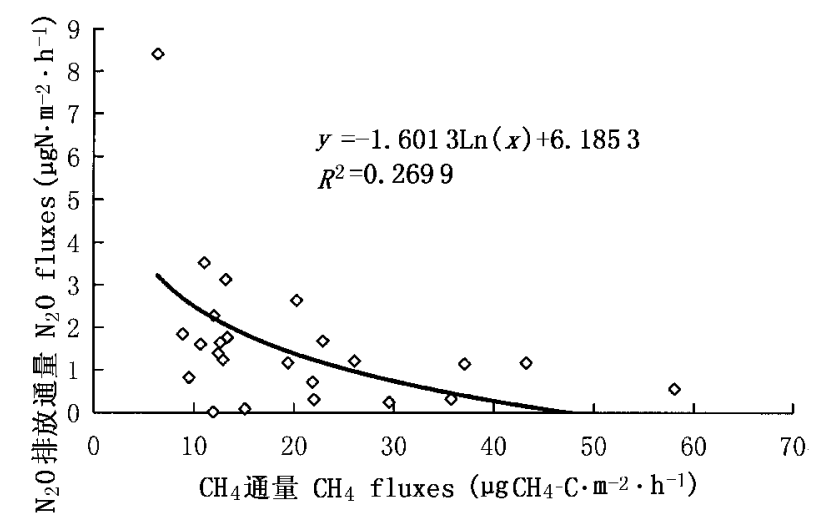

图 3 草甸草原土壤-植被系统 $\mathrm{N}_{2} \mathrm{O}$ 排放通量与 $\mathrm{CH}_{4}$ 吸收通量的关系 Fig.3 Relationship between $\mathrm{N}_{2} \mathrm{O}$ emission and $\mathrm{CH}_{4}$ consumption from soil-vegetation system in meadow steppe
1997), 这与硝化过程中氨的氧化反应类似, 只不过 后一过程由化能自养硝化细菌通过氨专性氧化酶 (AMO) 催化完成, 但是, 由于两类细菌在许多方面十 分相似 (Topp \& Pattey, 1997), 甲烷氧化也可通过 AMO 完成, 因此, 土壤中的铵与甲烷将竞争同一氧 化位点, 假定 $\mathrm{N}_{2} \mathrm{O}$ 主要产生于土壤硝化过程(Mosier et al ., 1991; 王艳芬等, 1997), $\mathrm{N}_{2} \mathrm{O}$ 的排放通量和 $\mathrm{CH}_{4}$ 的吸收通量均受土壤中 AMO 的影响而可能出 现此消彼长的情况。尽管如此, 由于这两个过程还 同时受到非生物因素的控制, 这种相关关系的存在 需要一定的环境条件, 例如, 在极干旱条件下, 土壤 排放 $\mathrm{N}_{2} \mathrm{O}$ 和吸收 $\mathrm{CH}_{4}$ 的速率都将降低 (Mosier et al ., 1991)。

\section{4 讨 论}

由于温室气体排放具有明显的昼夜变化规律, 时间变异性很大, 因此, 每个采样日采样时间的选择 十分重要,昼夜变化规律观测对确定每日采样时间 具有重要参考价值。目前国际上一般选择 9:00 12：00 作为当日采样时间 (Mosier et al., 1991;

Mosier \& Delgado, 1997; Mosier et al., 1993; Kessavalou et al . , 1998), 并假定此测定值能代表当 日交换通量, Epstein 等 (1998) 则建议在 10:00 14:00之间采取灵活的采样起始时间以防止总是观 测到交换通量高峰。

表 2 和表 3 分别给出了草甸草原 $\mathrm{CH}_{4}$ 和 $\mathrm{N}_{2} \mathrm{O}$ 日 平均地-气交换通量、10:00 和 13:00 时交换通量。 通过简单的比较可以发现, 尽管不同月份 10:00 和 13:00 的 $\mathrm{CH}_{4}$ 和 $\mathrm{N}_{2} \mathrm{O}$ 通量变化较大, 但是 10:00 时 $\mathrm{CH}_{4}$ 通量总比 13:00 时小, 而 $\mathrm{N}_{2} \mathrm{O}$ 通量前者总比后 者大, 这说明 $\mathrm{CH}_{4}$ 和 $\mathrm{N}_{2} \mathrm{O}$ 通量日变化规律强烈地受 到节律性变化因子的控制。 $\mathrm{CH}_{4}$ 昼夜变异系数为 $6.8 \% \sim 71.4 \%$, 这与 Mosier 等(1991)得出的 $\mathrm{CH}_{4}$ 变 化小于 $10 \%$ 的结果相比大得多。 $\mathrm{N}_{2} \mathrm{O}$ 的昼夜通量变 化则更大, 与其它研究中报道的结果比较一致

表 2 草甸草原 $\mathrm{CH}_{4}$ 日平均吸收通量与 10:00、13:00 瞬时通量的比较

Table 2 Diurnal mean $\mathrm{CH}_{4}$ uptake and instantaneous uptake on 10:00 and 13:00

\begin{tabular}{lccc}
\hline & $\begin{array}{c}\mathrm{CH}_{4} \text { 日平均通量 } \\
\text { Mean } \mathrm{CH}_{4} \text { daily }\end{array}$ & $\begin{array}{c}10: 00 \\
\mathrm{CH}_{4} \text { 通量 } \\
\mathrm{CH}_{4} \text { flux }\end{array}$ & $\begin{array}{c}13: 00 \\
\mathrm{CH}_{4} \text { 通量 } \\
\mathrm{CH}_{4} \text { flux }\end{array}$ \\
\cline { 2 - 4 } & \multicolumn{3}{c}{$\left(\mu \mathrm{CH}_{4}-\mathrm{C}^{-} \mathrm{m}^{-2} \bullet \mathrm{h}^{-1}\right)$} \\
\hline 6 月 June & $-52.19 \pm 19.67$ & -30.50 & -44.12 \\
7 月 July & $-27.20 \pm 10.57$ & -29.33 & -41.25 \\
8 月 August & $-12.05 \pm 9.32$ & -14.69 & -25.83 \\
9 月 September & $-16.46 \pm 1.32$ & -15.94 & -16.53 \\
\hline
\end{tabular}


表 3 草甸草原 $\mathrm{N}_{2} \mathrm{O}$ 日平均吸收通量与 10:00、13:00 通量的比较 Table 3 Diurnal Mean $\mathrm{N}_{2} \mathrm{O}$ flux and instantaneous flux on 10:00 and 13:00

\begin{tabular}{lccc}
\hline & $\begin{array}{c}\mathrm{N}_{2} \mathrm{O} \text { 日平均通量 } \\
\text { Mean } \mathrm{N}_{2} \mathrm{O} \text { daily }\end{array}$ & $\begin{array}{c}10: 00 \\
\mathrm{~N}_{2} \mathrm{O} \text { 通量 } \\
\mathrm{N}_{2} \mathrm{O} \text { flux }\end{array}$ & $\begin{array}{c}13: 00 \\
\mathrm{~N}_{2} \mathrm{O} \text { 通量 } \\
\mathrm{N}_{2} \mathrm{O} \text { flux }\end{array}$ \\
\cline { 2 - 4 } & \multicolumn{3}{c}{$\left(\mu \mathrm{g} \mathrm{N}_{2} \mathrm{O}-\mathrm{C} \cdot \mathrm{m}^{-2} \cdot \mathrm{h}^{-1}\right)$} \\
\hline 6 月 June & $0.282 \pm 0.802$ & 1.677 & -0.239 \\
7 月 July & $0.613 \pm 1.011$ & 0.308 & -0.576 \\
8 月 August & $2.134 \pm 2.766$ & 3.522 & 1.169 \\
9 月eptember & $1.492 \pm 1.209$ & 2.277 & 1.391 \\
\hline
\end{tabular}

（Slemr \& Seller, 1984)。由于 10:00 13:00 之间 $\mathrm{CH}_{4}$ 和 $\mathrm{N}_{2} \mathrm{O}$ 通量在整个生长季的变化是单向增加或 单向减少的, 因此可将 10:00 和 13:00 时的通量视 为一个区间的两个端点, 如果把 $\mathrm{CH}_{4}$ 和 $\mathrm{N}_{2} \mathrm{O}$ 日平均 通量落入该区间与否作为判断 10:00 13:00 之间 测得的通量是否能够代表当日通量的标准, 那么从 整个生长季来看, 10:00 13:00 的测定结果很具有 代表性。

因此, 在内蒙古草原选择 9:00 13:00 作为每 个采样日的采样时间是比较合适的。

\section{参 考 文 献}

Bronson, K. F. \& A. R. Mosier. 1993. Nitrous oxide emissions and methane consumption in wheat and corn-cropped systems. In: Harper, L. A. ed. Agricultural ecosystem effects on trace gases and global climate change. Madison: American Society of Agronomy Special Publication. 55: $133 \sim 144$.

Bronson, K. F., A. R. Mosier \& S. R. Bishnoi. 1992. Nitrous oxide emissions in irrigated corn as affected by encapsulated calcium carbide and nitrapyrin. Soil Science Society of America Journal, 56: $161 \sim 165$.

Conrad, R. 1996. Soil microorganisms as controllers of atmospheric trace gases $\left(\mathrm{H}_{2}, \mathrm{CO}, \mathrm{CH}_{4}, \mathrm{OCS}, \mathrm{N}_{2} \mathrm{O}\right.$ and $\left.\mathrm{NO}\right)$. Microbiology Review, 60:609 640.

Corre, M. D., D. J. Pennock, C. van Kessel \& D. K. Elliott. 1999. Estimation of annual nitrous oxide emissions from a transitional grassland-forest region in Saskatchewan, Canada. Biogeochemistry, 44:29 49.

Davidson, E. A. \& J. P. Schimel. 1995. Microbial processes of production and consumption of nitric oxide, nitrous oxide and methane. In: Matson, P. A. \& R. C. Harriss eds. Biogenic trace gases: measuring emissions from soil and water. Malden, Mass: Blackwell Science. $327 \sim 357$.

Dong, Y.S. (董云社), S. Zhang (章申), Y.C. Qi (齐玉春), Z.Z. Chen(陈佐忠) \& Y.B. Geng (耿元波). 2000. Fluxes of $\mathrm{CO}_{2}, \mathrm{~N}_{2} \mathrm{O}$ and $\mathrm{CH}_{4}$ from a typical temperate grassland in Inner Mongolia and its daily variation. Chinese Science Bulletin, 45: $318 \sim 322$.

Du, R. (杜睿)，G.C. Wang (王庚辰)，G.R. Liu (刘广仁)， Y.F. Wang(王艳芬)，Y.S. Wang(王跃思)，D. R. Lü(吕 达仁) \& Z. Z. Chen(陈佐忠). 1998. The study on diurnal variation in greenhouse gas revenue and expenditure fluxes of Leymus chinensis grassland of Inner Mongolia. Acta Agrestia Sinica(草地学报), 6: $258 \sim 264$. (in Chinese with English abstract)

Epstein, H.E., I.C. Burke, A.R. Mosier \& G. L. Hutchinson.
1998. Plant species effects on trace gas fluxes in the shortgrass steppe. Biogeochemistry, 42:145 168 .

Groffman, P. M. \& C. L. Turner. 1995. Plant productivity and nitrogen gas flux in a tallgrass prairie landscape. Landscape Ecology, 10: $255 \sim 266$.

Ji, B.M. (纪宝明), Y. F. Wang (王艳芬), X.Z. Li (李香 真), Y.S. Wang (王跃思), Z.Z. Chen (陈佐忠) \& X. H. Zheng(郑循华). 2001. $\mathrm{CH}_{4}$ and $\mathrm{CO}_{2}$ concentration in soils of the major grasslands of the Xilin River Basin of Inner Mongolia. Acta Phytoecologica Sinica (植物生态学报), 25:371 374. (in Chinese with English abstract)

Kessavalou, A., A.R. Mosier, J. W. Doran, R. A. Drijber, D. J. Lyon \& O. Heinemeyer. 1998. Fluxes of $\mathrm{CO}_{2}, \mathrm{~N}_{2} \mathrm{O}$ and $\mathrm{CH}_{4}$ in grass sod and winter wheat-fallow tillage management. Journal of Environmental Quality, 27: 1094 1104 .

Luo, J., R. W. Tillman \& P. R. Ball. 1999. Grazing effects on gentrification in a soil under pasture during two contrasting seasons. Soil Biology \& Biochemistry, 31: $903 \sim 912$.

Mosier, A., D. Schimel, D. Valentine, K. Bronson \& W. Parton. 1991. Methane and nitrous oxide fluxes in native, fertilized and cultivated grasslands. Nature, 350:330 332 .

Mosier, A.R. \& J.A. Delgado. 1997. Methane and nitrous oxide fluxes in grasslands in western Puerto Rico. Chemosphere, 35: $2050 \sim 2082$.

Mosier, A. R., L. K. Klemedtsson, R. A. Sommerfeld \& R. C. Musselman. 1993. Methane and nitrous oxide flux in a Wyoming subalpine meadow. Global Biogeochemical Cycles, 7: 771 784.

Mosier, A.R., W.J. Parton, D.W. Valentine, D.S. Ojima, D. S. Schimel \& J.A. Delgado. 1996. $\mathrm{CH}_{4}$ and $\mathrm{N}_{2} \mathrm{O}$ fluxes in the Colorado shortgrass steppe. I . Impact of landscape and nitrogen addition. Global Biogeochemical Cycles, 10:387 399 .

Slemr, F. \& W. Seller. 1984. Field measurements of $\mathrm{NO}$ and $\mathrm{NO}_{2}$ emissions from fertilized and unfertilized soils. Journal of Atmospheric Chemistry, 1:159 169.

Smart, D. R., J. M. Stark \& V. Diego. 1999. Resource limitations to nitric oxide emissions from a sagebrush-steppe ecosystem. Biogeochemistry, 47: $63 \sim 86$.

Topp, E. \& E. Pattey. 1997. Soils as sources and sinks for atmospheric methane. Canadian Journal of Soil Science, 77:167 178 .

Wang, G. C. (王庚辰), R. Du (杜睿), Y. F. Wang (王艳 芬), G. R. Liu(刘广仁), Y. S. Wang (王跃思), Z. Z. Chen(陈佐忠) \& D. R. Lü(吕达仁). 1998. Characteristics of $\mathrm{N}_{2} \mathrm{O}$ and $\mathrm{CH}_{4}$ fluxes and their seasonal variations from Inner Mongolia grassland. Acta Agrestia Sinica (草地学报), 6: 306 311. (in Chinese with English abstract)

Wang, Y. F. (王艳芬), Z. Z. Chen (陈佐忠) \& L. T. Tieszen. 1998. Distribution of soil organic carbon in the major grasslands of Xilingole, Inner Mongolia, China. Acta Phytoecologica Sinica (植物生态学报), 22:545 551. (in Chinese with English abstract)

Wang, Y.F. (王艳芬), Z.Z. Chen(陈佐忠) \& Y. Zhou(周 涌). 1997. Studies on $\mathrm{N}_{2} \mathrm{O}$ emission from typical steppe in Inner Mongolia. Climatic and Environmental Research (气候与环 境), 2: $280 \sim 285$. (in Chinese with English abstract)

Wang, Y.S. (王跃思), B.M. Ji (纪宝明), Y.F. Wang (王艳 芬), W. Zhang (张文), G. R. Liu (刘广仁), R. Du (杜 点) \& D. M. Luo (骆冬梅). 2000. Measurement of the exchange rate of greenhouse gases between field and atmosphere in semi-arid grassland. Environmental Science (环境科学) , 3:6 10. (in Chinese with English abstract)

Williams, E. J., G. L. Hutchinson \& F. C. Fehsenfeld. 1992. NOx and $\mathrm{N}_{2} \mathrm{O}$ emission from soil. Global Biogeochemical Cycles, 6:351 388 . 